\title{
INDIVIDUAL DIVERSITY AND TEMPORAL STABILITY DURING A 4-MONTH CONFINEMENT EXPERIMENT (SIRIUS-19) FOR HUMAN SPACE EXPLORATION
}

\author{
Carole Tafforin', Alla Vinokhodova ${ }^{2}$, Vadim Gushin² \\ ${ }^{1}$ Research and Study Group in Human and Space Ethology (Ethospace), Toulouse, France \\ ${ }^{2}$ Institute of Bio-Medical Problems of RAS (IBMP), Moscow, Russia
}

ethospace@orange.fr

\begin{abstract}
With a view to space colonization on the scale of evolution, travels to the Moon then to Mars are the near future of human exploration. New missions scenarios involve isolated and confined crews with heterogeneous composition and autonomous organization on long-duration experiences. SIRIUS-19 paradigm offered the opportunity of studying the behavioral adaptation of 6 crewmembers (3 women of Russian nationality, 1 Russian man and 2 men of American nationality) over a 133-day experiment in confinement conditions. The protocol simulated major milestones of a Moon mission for an isolation lasting 120 days. We used the ethological method based on qualitative and quantitative analyses of non-verbal and verbal behaviors from video recordings collected at breakfast time twice a month and during a group discussion once a month. The results showed differences between subjects in the frequency profiles of interactions, motions, expressions and communications on specific mission days, and a mean linear trend with no significant correlation according to time. Individual diversity with positive behavioral strategies and temporal stability with no third-quarter phenomenon are discussed. We concluded that a salutogenic adaptive step has been taken in the development of the social group.
\end{abstract}

Keywords: Adaptation, Salutogenesis, Social group, Human settlement, Isolated and Confined Environment

Acronyms: ICE (Isolated and Confined Environment), SIRIUS (Scientific International Research in Unique Terrestrial Station), International Space Station (ISS), TQP (Third-Quarter Phenomenon), BF (Breakfast), GD (Group Discussion), MD (Mission Day), EVA (Extra-Vehicular Activity). 


\section{INTRODUCTION}

Whilst Earthlings are experiencing today Earth isolation and confinement conditions (Sharma, 2020; Vermaa et al., 2020), humankind will explore them tomorrow as a new environment of behavioral adaptation. During long duration missions for planets exploration, the isolated and confined crews will have to anticipate, to try, to compensate, to change, to innovate, to build and to secure as positive adaptive strategies for obtaining salutogenic effects rather than anxiety-provoking ones. Salutogenesis, i.e. having a positive effect for persons undergoing challenging experiences in extreme environments, was first reported about individual and group adaptation in Antarctica (Palinkas, 2003). It was stated that stress could actually be beneficial, be health-promoting and bring improvements in performance and well-being. Behavioral health implies feeling good about oneself, and rewarding interactions with the physical environment (Harrison \& Fiedler, 2010). Lessons from a series of studies in a space simulator, onboard the Mir station and the International Space Station (ISS), supported the idea that spaceflights and analog settings can provide mental health benefits, even though the experiences may be fraught with danger, hard work, social and familial distancing (Ritsher et al., 2007). As salutogenic changes, cosmonauts and astronauts developed coping strategies (Suedfeld et al. 2015). When we push the boundaries of human experience, time becomes a core component of future manned missions. The permanent establishment of a Moon village (Foing, 2016) and a journey to explore Mars with 2-year or 3-year free return (Wooster et al., 2007) and beyond (Smith, 2016) are not mission scenarios as we have known until now. They will be novel in many ways (Sherwood, 2017, Marboe, 2019). We are in the near future when considering interplanetary flights within ten to thirty years but we are in the distant future when imagining space colonization on the scale of evolution.

\section{The planetary environment}

Discussions of exploration of the red planet raised controversies, such as whether humans should and could not go to Mars (Szocik, 2019). The fact of being a multi-planet species has also been raised. Would humanity be prepared (Marino, 2019) or should we first gain more experience and knowledge before colonizing outer space in order to mitigate risks (Slobadian, 2015)? One reply is that it could be the only long-term option for Earthlings' survival (Cirkovic, 2019). But we know that human beings are an intelligent species in terms of adaptation capabilities and creative abilities. Once on Mars, human factors such as leadership structure, social organization, codes of conduct, group size, gender balance, development cycle, ecological settings and manner of subsistence, will create a novel Martian culture (Szocik et al., 2020). Medium to long-term stays in novel, extraordinary or analog environments present time factors to consider. In space ethology, we analyze relationships between the individual and the extreme environment as optimization processes and we follow temporal dynamics since positive adaptive steps are needed.

\section{Individual level}

Previous studies began with investigations of crewmembers' behavior during extended periods of isolation and confinement that prefigure new missions to the Moon and future travels to Mars and back on Earth. Individual behavior is the first indication of the wellbeing and good morale of subjects operating within social constraints and spatial restraints (Tafforin, 2018). Related psychological researches have addressed personal values (Ma et al., 2019), personality traits (Mittelstäedt el al. 2016), personal growth (Solcovà \& Tavel, 2017), personal attitudes (Vinokhodova \& Gushin, 2014), emotional states (Polackova, Lacey \& Solcovà, 2014), communicative profiles (Gushin et al., 2012), and mental health, 
with the aim of identifying individual characteristics indicating strong motivation (Kanas et al., 2009). Some characteristics of personality, such as conscientiousness, agreeableness, extraversion and neuroticism are linked to performance and would play a role in the degree of adaptation (Rosnet et al., 2000 and Palinkas et al., 2000 cited in Collado et al. 2012). Examining personal values in extreme environment contexts, such as populations of mountaineers, military personnel, Antarctic over-winterers and Mars simulation participants, showed rank scores according to Schwartz's basic values definition (Schwartz et al., 2012). These groups were comparable on self-direction, stimulation, benevolence, tradition and security (Smith et al., 2017) but differences that emerged on certain values emphasized the importance of considering them in specific compositing crews for long duration missions (Sandal et al., 2011).

Between-person differences have to be investigated so as to have a beneficial effect on crews' adaptation over time.

\section{Temporal level}

Time-course changes in individual and inter-individual behaviors have been studied in various Isolated and Confined Environments (ICEs). In the previous studies, ethological indicators were monitored in small groups living and working together inside confinement chambers during three experimental campaigns of different duration. The Isolation Study of European Manned Space Infrastructure (ISEMSI) experiment was of 1-month duration, the EXperimental campaigns for European Manned Space Infrastructure (EXEMSI) was of 2month duration, and the Human Behavior in Extended Spaceflight (HUBES) was of 4month duration. Results showed that the frequency of personal distances decreased and the frequency of public distances increased, with higher levels of social distance and body mobility from the initial period to the final period of the simulation missions (Tafforin, 2005). Mid-period was marked by critical temporal points. Similar behavioral profiles were observed in a polar team at the Dumont d'Urville Antarctic station over an 8-month isolation (Tafforin, 2004). Behavioral changes occurred independently of the mission duration except for period-dependent differences that emphasized the so-called ThirdQuarter Phenomenon (TQP) ranging from Antarctica to outer space. The third quarter is just after the mid-point, when the end is in sight, characterized by increased homesickness, depression, irritability and decreased crew cohesion (Betchel \& Berning, 1991). Recent researches have found decrements in performance in ICEs that support the TQP associated to psychological resilience of winterers at the Concordia Antarctic station (Sandal, Van DeVijver \& Smith, 2018) and to coping strategies of submariners in underwater deployment (Van Wijk, 2018). Nevertheless, during other confinement experiments conducted in the Lunar Palace 1 analogue space station, findings on crewmembers' mood profiles did not support the TQP (Wu \& Wang, 2015). An open social network inside the chamber, daily physical exercise and full support from outside may have been beneficial in the late mission stage (Wang \& Wu, 2015).

Group dynamics have to be considered in a stage-model of adaptation.

\section{The dependent variables}

Behavioral analysis of a crew's collective activities is of prime interest for identifying the salutogenic adaptive strategies built over time. Our theoretical hypothesis is: individual diversity and temporal stability are key factors of such strategies that contribute to sociopsychological health, and help to optimize the relationship between the individual and the environment. Human factors act in synergy with time factors for mission success. Our working hypothesis is: different levels of subjects' interactions, expressions, movements and communications mitigate negative effects of a 4-month confinement. Low variations over 
time indicate positive effects of these behaviors in the social group. The Scientific International Research in Unique Terrestrial Station (SIRIUS) program offers an opportunity for studying these effects.

\section{METHODS}

\section{The SIRIUS-19 experiment}

SIRIUS-19 took place from 19th March 2019 to 17th July 2019 at the Institute of Biomedical Problems (IBMP) in Moscow, Russia. The experiment was completed in the Ground Test Complex (NEK) used for isolation and confinement paradigms of different duration. It is a multi-chamber facility of 5 units: EU-50 landing module, EU-100 working module, EU-150 living module, EU250 storage module and PSS planetary surface simulator.

\section{Subjects}

The crew was composed of 6 subjects equally comprising three males (subjects M1, M2, M3) and three females (subjects F4, F5 and F6), with four Russians and two Americans. They were between 28 and 44 years old. Roles during the experiment were one commander, one flight engineer, one crew physician and three researchers. In addition to daily health checks and medical examinations, the subjects made investigations on their own to study the effects of such extreme conditions on human physiology and psychology for space colonization scenarios.

All the crew members gave their informed consent before the experiment started.

\section{Milestones}

The experimental goal was to follow the scenario of an actual Moon mission (Figure 1). Milestone 1: the crew starts for the Moon, arrives at the orbit and docks to an orbital station (e.g. Gateway). Milestone 2: for the period of two months, the crew conducts observations of the lunar surface in order to select the landing site, and performs several docks with arriving transport vehicles. Four subjects landed on the Moon where two of them performed extra-vehicular activities (EVA). They then returned to the orbiter. Milestone 3: over several weeks the crew orbits the Moon in order to remotely operate Moon rovers and to simulate the foundation of lunar settings. Upon completion of the milestones, the crew returns to Earth after 133 days in ICE.

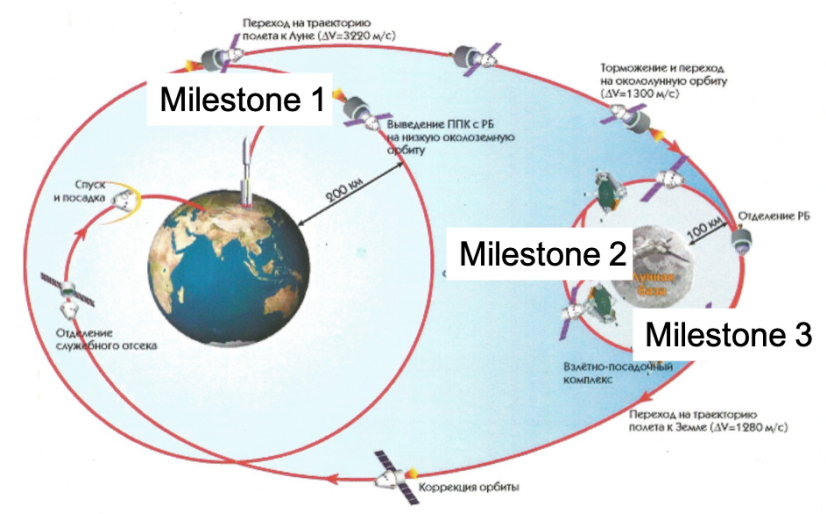

Figure 1: SIRIUS-19 Scenario. Image credits : SSC RF-IBMP RAS and HPR NASA. 


\section{The ethological approach}

\section{Method}

We used methodological tools from human ethology, based on observation, description and quantification of the behavioral manifestations visualized in living and working situations (e.g. meal time, given tasks). Qualitative analysis results from encoding objective events based upon a list of behavior units categorized as actions, interactions and communications. In the present study, state events (duration $>3 \mathrm{~s}$.) are personal actions (any body segment movements, postural changes), visual interactions (any regards directed to one subject), body interactions (any body contacts between two subjects), object interactions (any manipulations from one subject to the other one), body mobility (motions in the main living area) and verbal interactions (oral communications between the subjects). Point events (duration < 3s.) are facial expressions (as smiling, laughing), body expressions (any body segment movements as body language) and collateral acts (any small movements with no manifested functions such as scratching the head, scratching the nose).

The subjects' behavior was scored with The Observer $\mathrm{XT}^{\circledast}$ 14.0. Quantitative analysis results from non-parametric descriptive statistics.

\section{Protocol}

The advanced logistic support is the subjects' video recording collected with four safety cameras intersecting the total observational area of the observations situations, completed with one fisheye camera. Twice a month, video recordings were performed at breakfasts $(\mathrm{BFs})$, in the kitchen of the living module. Data collection protocol was on nine temporal points (MD5, MD20, MD34, MD48, MD62, MD76, MD89, MD118, MD132). Once a month, video recordings were performed during group discussions (GDs), from the same cameras set. Data collection protocol was on four temporal points (MD17, MD48, MD101, MD128).

BF living situation was a free collective activity and was chosen for analyzing the spontaneous non-verbal behaviors (interactions, expressions, motions) of the crewmembers in a daily life activity according to the Mission Day (MD). We analyzed various 15- to 30minute time slots while subjects were in a sitting position around the BF table. GD working situation focused on an imposed topic to bring together all the crewmembers and generated verbal behaviors (communications) between them. We analyzed equal duration recordings (15 minutes) when subjects started speaking after a reading period about the topic of the day.

One methodological limitation is possible failure to keep in the field of the observable events for objective analyses and data standardization. Total BF observations were 23 hours (Mean duration $=26$ minutes; $\mathrm{SD}=5$ ). Total GD observations (Lowest Common Denominator duration $=15$ minutes) were 6 hours ( 15 minutes $\mathrm{x} 4$ days $\mathrm{x} 6$ subjects).

\section{RESULTS}

\section{Interactions}

In Figure 2, the interactions profile is presented for each subject at breakfast over the mission days. The relative frequencies of this behavioral category, including visual interactions, body interactions and object interactions, vary between 0 and 2.6 acts per minute. Variation dynamics are globally similar in all the subjects. We observed an increase 
of occurrences from MD5 to MD20, a decrease on MD34, increases on MD62 and MD76, and an increase on MD132. The results show a linear trend with low significant correlation $(\mathrm{p}<0.5)$ of the mean frequencies according to time. That showed that interactions of the subjects exhibited temporal stability. However, there are some specific temporal points in the individual profiles. We observed behavioral differences in subject M3, subject F5 and subject F4, on MD20, MD34 and MD118, respectively.

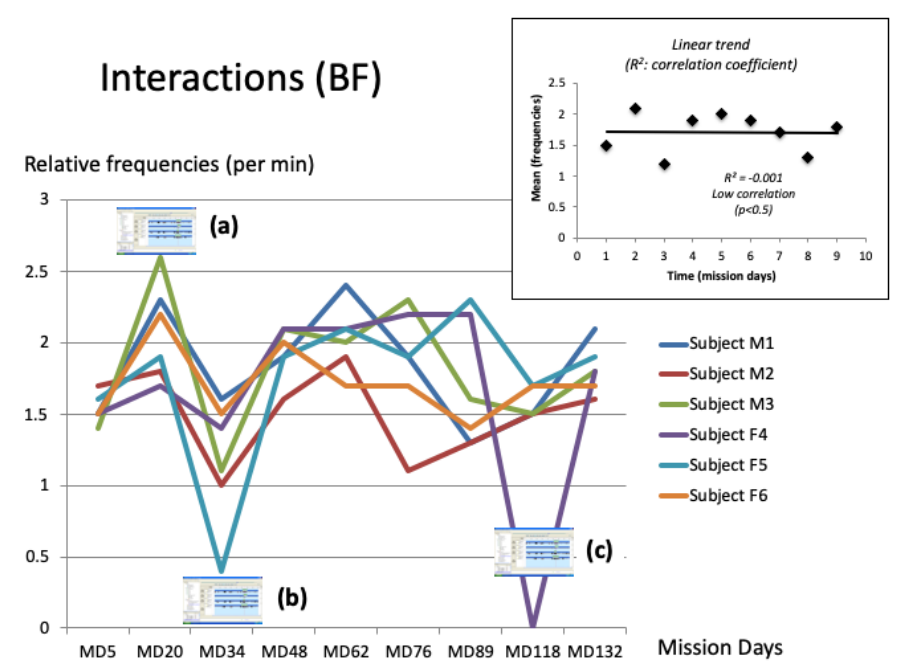

Figure 2: Occurrences in relative frequencies of the subjects' interactions according to the mission days during a 4-month confinement (SIRIUS-19). Statistics: correlation coefficient $\left(R^{2}\right)$ of mean frequencies over time.

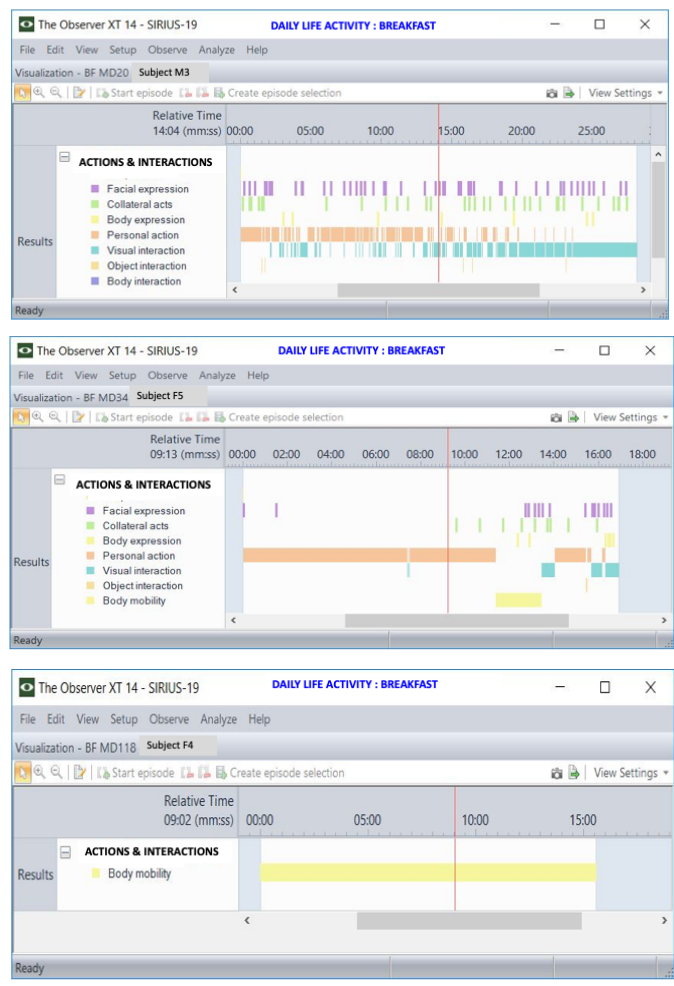

Figure 2a: Behavioral data visualization of subject M3, on mission day 20, at breakfast (BF).

Figure 2b: Behavioral data visualization of subject F5, on mission day 34, at breakfast (BF).

Figure 2c: Behavioral data visualization of subject F4, on mission day 118, at breakfast (BF). 
In Figure 2(a), data visualization of subject M3 on MD20 offers an overview of his nonverbal behavior, including body interactions, object interactions, visual interactions, personal actions, body expressions, collateral acts and facial expressions, over the whole breakfast duration. In half of the period, visual interactions were particularly steady and frequent in that subject.

In Figure 2(b), data visualization of subject F5 on MD34 offers an insight on what happened specifically on this mission day. We observed very low levels of interactions vs. personal actions. By the end the activity, visual interactions occurred at the same time as facial expressions. This behavioral profile occurred in all the subjects.

In Figure 2(c), data visualization of subject F4 on MD118 offers another example of individual behavior that is unusual at breakfast when all the subjects are usually in sat positions around the collective table. We observed long duration body mobility throughout the activity. Motions outside the kitchen decreased any interactions as a result.

\section{Motions}

In Figure 3, the motions profile is presented for each subject at breakfast over the mission days. The relative frequencies of this behavioral category, including any position changes in the dinning room, vary between 0 and 0.3 acts per minute. The most constant level from MD5 to MD132 is 0.1 acts per minute. We observed low levels of motions that maintain the subjects together during this collective activity all over the mission. The results show linear trend with no significant correlation according to time and reveal a temporal stability of social behavior.

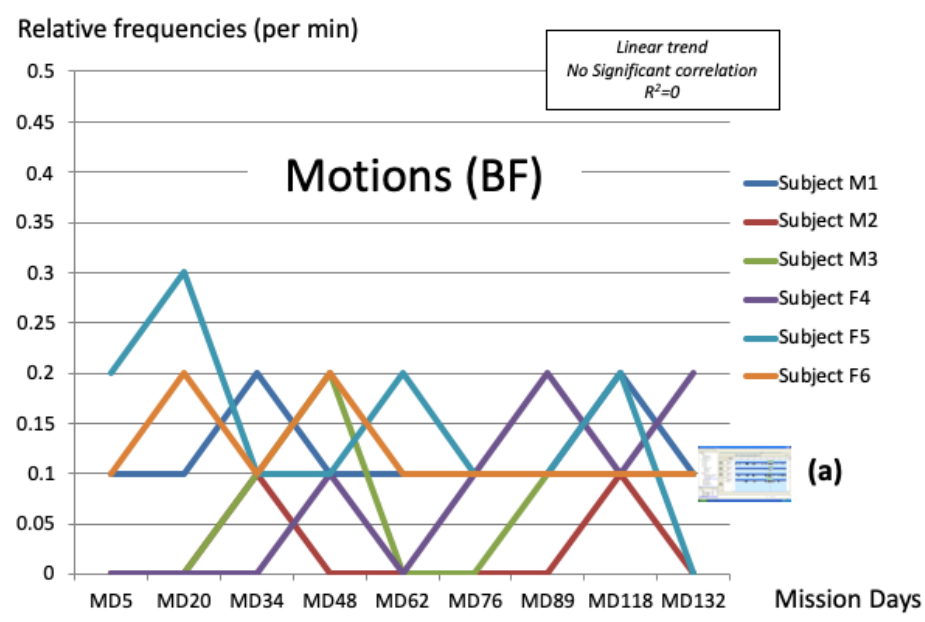

Figure 3: Occurrences in relative frequencies of the subjects' motions according to the mission days during a 4-month confinement (SIRIUS-19). Statistics: correlation coefficient $\left(\mathrm{R}^{2}\right)$ of mean frequencies over time.

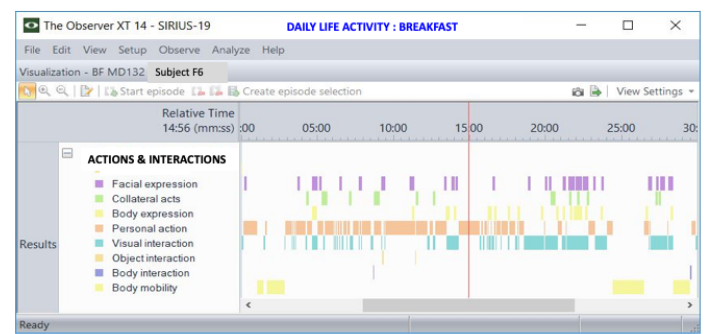

Figure 3a: Behavioral data visualization of subject F6, on mission day 132, at breakfast (BF). 
In Figure 3(a), data visualization of subject F6 on MD132 offers an overview of her nonverbal behavior after a 4-month confinement. Well-being facial expressions were frequent but stress-related collateral acts still occurred. Personal actions and visual interactions were the most prolonged whereas object interactions and body interactions were at the lowest levels. There were sometimes body mobility and body expressions intermittently. This behavioral profile appears as a wide pattern of actions and interactions, an indicator of a positive attitude by the end of the mission.

\section{Expressions}

In Figure 4, the expressions profile is presented for each subject at breakfast over the mission days. The relative frequencies of this behavioral category, including facial expressions and body expressions, vary between about 0 and 5 acts per minute, with occurrence peak on MD132. Subject F5 was at the highest level overall. She had an expressive behavior, and acted positively at the end of the mission, like subject F6. The results show a linear trend with low significant correlation $(\mathrm{p}<0.5)$ of the mean frequencies over time. That showed the expressions exhibited temporal stability.

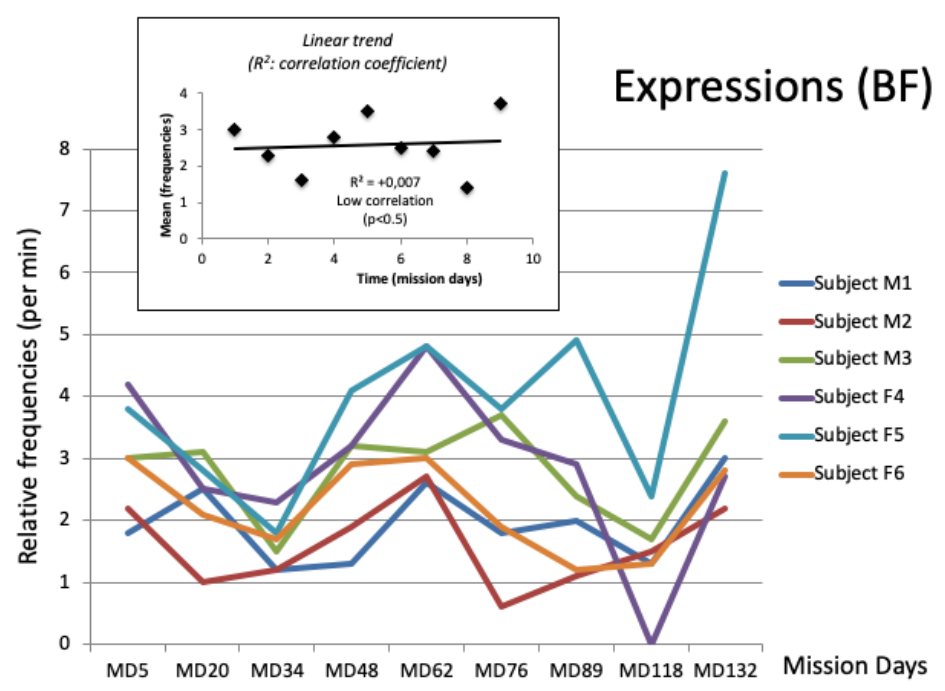

Figure 4: Occurrences in relative frequencies of the subjects' expressions according to the mission days during a 4-month confinement (SIRIUS-19). Statistics: correlation coefficient $\left(\mathrm{R}^{2}\right)$ of mean frequencies over time.

\section{Communications}

In Figure 5, the communications profile is plotted for each subject during group discussions over the mission days. The highest level is on MD101 (174 acts over 15-minute task duration) and the lowest level is on MD128 (102 acts over 15-minute task duration). We observed individual differences. The absolute frequencies of this behavioral category decreased significantly in subject M1 $(\mathrm{p}<0.01)$ and in subject F6 $(\mathrm{p}<0.1)$ from the beginning to the end of the mission. Subject M2 was infrequently involved in communications, subject M3 varied the levels alternately, subject F4 was constant and subject F5 was the most communicative from MD17 to MD128. 


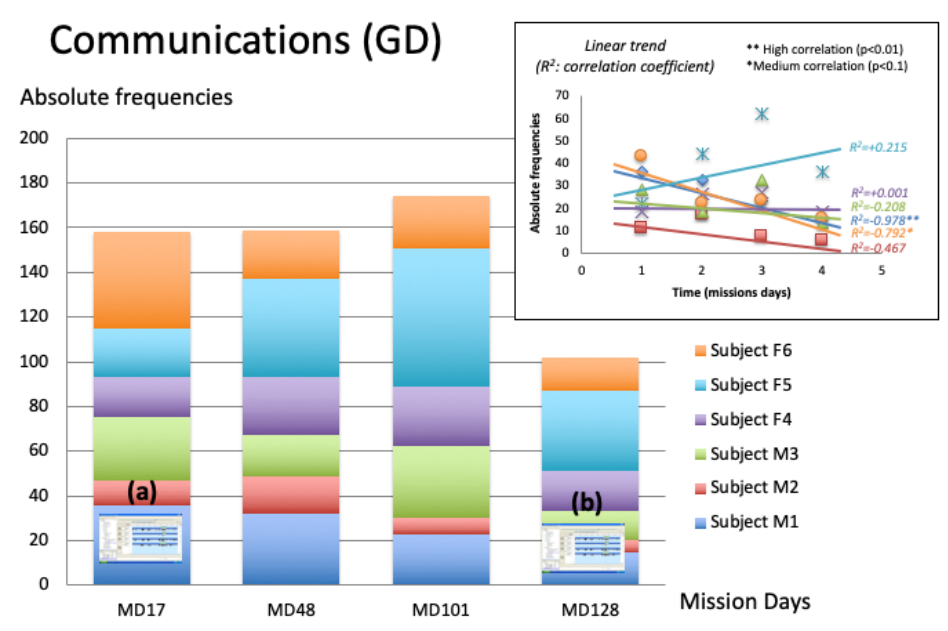

Figure 5: Occurrences in relative frequencies of the subjects' communications according to the mission days during a 4-month confinement (SIRIUS-19). Statistics: correlation coefficient $\left(\mathrm{R}^{2}\right)$ of mean frequencies over time.
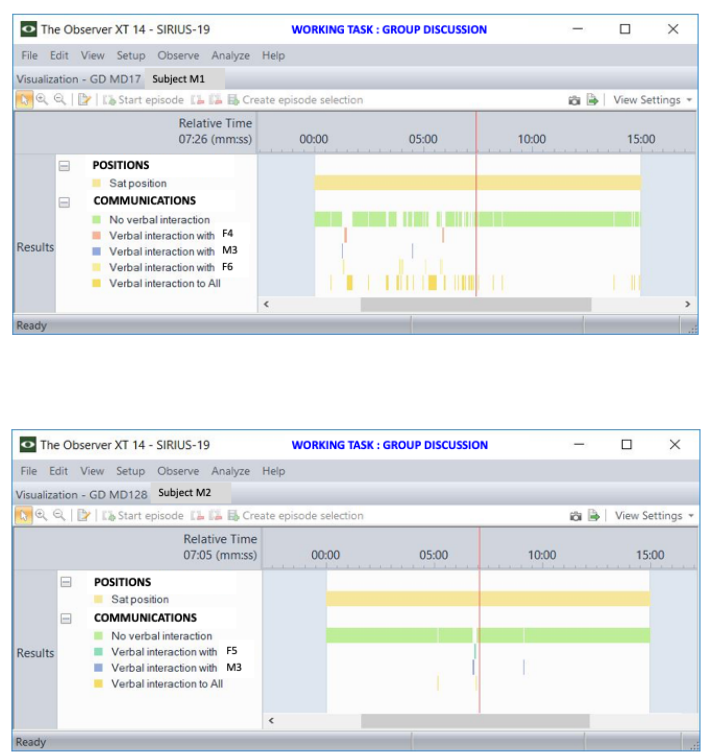

Figure 5a: Behavioral data visualization of subject M1, on mission day 17 , during the group discussion (GD).

Figure 5b: Behavioral data visualization of subject M2, on mission day 128 , during the group discussion (GD).

In Figure 5(a), data visualization of subject M1 on MD17 offers a qualitative overview of his verbal behavior during a collective task (GD) after a 4-month confinement. The results show that he communicated frequently within the first half of the GD, including a reading period of the topics and a speaking period that initiated the discussion. There were few verbal interactions with subjects individually but numerous verbal interactions with the group. Then we observed a long duration of no verbal interaction.

In Figure 5(b), data visualization of subject M2 on MD128 offers an overview of his verbal behavior during a collective task (GD) after the 4-month confinement. The results show that he was not communicative at all, by the end of the mission. We observed a long duration of no verbal interaction over the whole task, with some episodic communications about the half time. 


\section{DISCUSSION}

The first step to go to Mars will be to go on the Moon with transfers by the Gateway station. The SIRIUS program is an exceptional paradigm for such manned mission scenarios. From a behavioral viewpoint, the subjects developed adaptive strategies during the SIRIUS-19 experiment and the mission was successful. In the ethological approach, we did not analyze the results of the behaviors that are performed, but rather the actions pattern leading to them: interactions, expressions, motions and communications. These events occurred in a positive way through individual diversity and temporal stability. Levels of frequency over time yield quantitative and correlative analyses. Data visualization offers a behavioral picture of the subject at a given time from a qualitative analysis.

\section{Individual diversity}

The SIRIUS-19 crew was a heterogeneous group with mixed gender, bi-cultural and multifunctional characteristics. Such diversity is involved in the crewmembers' behavioral adaptation. We previously had found expressive and communicative behaviors in female subjects, and less active (personal actions) but more interactive (inter-personal actions) behaviors in male subjects, with specific strategies built by each one during the 4-month confinement (Tafforin, 2020). The present study showed individual differences. Subject M1 engaged in frequent verbal interactions with all others during the GD, which stimulated communications between individuals. Then frequencies significantly decreased until the last $\mathrm{MD}$, to leave them spontaneously talking together, depending on their personality traits and personal values. Subject F5 was the most expressive at BF and most communicative during GD all through the mission. Facial expressions and body expressions were frequent, but with a sharp decrease of interactions on one day after one month of living and working together (MD34). The crew acted as if it had intentionally decided to perform personal actions vs. inter-personal actions at that time. Then visual interactions (gaze directed to the subjects) and facial expressions (smiling, laughing) resumed again by the end of breakfast time, emblematic of group cooperation. Subject F6 was particularly active, exhibiting diverse non-verbal behavioral events (facial expressions, body expressions, collateral acts, visual interactions, object interactions, body interactions and body mobility) after 4 months spent in ICE (MD132), whereas her verbal interactions (communications) significantly decreased over time. Conversely, subject M3 had the same profile of high interactions vs. actions at the beginning of the mission (MD20). This highlights synergies of human factors and time factors. One day (MD118), subject F4 decided idiosyncratically to move from the collective area (kitchen) to the living room for BF. Her adaptive strategy was to break up the monotonous conditions of daily life activities. Body mobility was, however, not important since collective attendance was required at meal time. Subject M2 was less involved in interactions, motions, expressions and communications.

As crew autonomy grew over the long-duration adaptation, between-person differences contributed to individual diversity by a sharing of general values (Vinokhodova and Gushin, 2014) and apparent self-organization. In this self-organized group characterized by the intrinsic quality of its elements (diversified composition of members, diversified experiences), personality factors facilitated relationships with the environment, be it physical, social or psychological. 


\section{Temporal stability}

The group dynamics of SIRIUS-19 evolved step by step in accordance with external factors. Milestones simulating the Moon mission scenario with various virtual and real operations outside the habitat (observations and search for a landing site, extra-vehicular activities, rover manipulations on the lunar surface, several docks with arriving transport vehicles) kept the crew in rhythmic workload conditions. This mitigates the stressful isolation and confinement conditions exacerbated over time, thus explaining the temporal stabilities in the crew's behavior. Regarding interactions, motions and expressions, there were no significant positive or negative correlations between non-verbal behaviors and mission duration. Within this 4-month period, there was no evidence of TQP as a result. We found individual changes according to the $\mathrm{MD}$, with specific temporal points, but the global changes were stable as crewmembers displayed similar dynamics of behaviors, with an emphasis on the interactions variation in all the crewmembers. We may consider the crew as acting in a time slice that is an adaptive step, and changes of behaviors could be an anticipatory process of moving on to another step.

Relationships between psychological stability and peculiarities of interactions in isolated and confined crews were considered with an eye toward the success of future Mars exploration missions (Kuznetsova et al., 2018). Emotional stability, or low anxiety, is a relevant indicator of a positive relationship between the individual and the social environment. It is a core factor for joint work and communications leading to crew cohesion. Ethological observations of verbal behaviors that showed temporal stability support this optimization in the relationship and the extent to which SIRIUS-19 crew was an adapted social group.

\section{Conclusion}

Observation, description and quantification of non-verbal and verbal behaviors in a daily life activity (BF) and during a collective task (GD) support our working hypothesis on different levels of subjects' interactions, expressions, motions and communications that mitigate negative effects of a 4-month confinement. Low variations over time indicate positive effects of these behaviors for the isolated and confined crew, and a salutogenic adaptive step.

Next correlative studies between ethological data and psychological data will consolidate the SIRIUS-19 findings on social cohesion. The next stages in the framework of the SIRIUS program regarding 8-month and 12-month confinements will be investigated. Additional topics might be investigated such as how the emotional life of the crew differed from that of people in less confined conditions or other terrestrial contexts. Shaped by evolutionary dynamics, multi-cultures and micro-societies travelling to far-off destinations from Earth are new research goals for human space exploration.

The authors declare that there is not conflict of interest.

\section{ACKNLOWEDGEMENTS}

The French Space Agency (CNES) financially supported this research. We thank the team of SIRIUS-19 experiment. 


\section{REFERENCES}

Betchtel, R.B., \& Berning, A. (1991). The third-quarter phenomenon: do people experience discomfort after stress has passed? In A.A. Harrison, Y.A. Clearwater, \& C.P. McKay (Eds). From Antarctica to outer space: Life in Isolation and confinement (pp. 261-265). New York: Springer Verlag. DOI

Cirkovic, M.M. (2019). Space colonization remains the only long-term option for humanity: a reply to Torres. Futures, 105, 166-173. DOI

Collado, A., Willmann, M., Caillet, G., Hainaut, J.P., \& Bolmont, B. (2012, October). Adaptation to parabolic flights. Poster ID 15100 presented at the 63rd International Astronautical Congress, Naples, Italy.

Foing, B. (2016, September). Towards a Moon village: enabling technology and precursor missions. Paper ID 35696 presented at the 67th International Astronautical Congress, Guadalajara, Mexico.

Gushin, V., Shved, D., Vinokhodova, A., Vasylieva, G., Nitchiporuk, I., Ehmann, B., \& Balazs, L. (2012). Some psychophysiological and behavioral aspects of adaptation to simulated autonomous Mission to Mars. Acta Astronautica, 70, 52-57. DOI

Harrison, A.A., \& Fiedler, E.R. (2010). Mars, human factors and behavioral health. Journal of Cosmology, 12, 3685-3693.

Kanas, N., Sandal, G., Boyd, J.E., Gushin, V.I., Manzey, D., North, R., Leon, G.R., Suedfeld, P., Bishop, S., Fiedler, E.R., Inoue, N., Johannes, B., Kealey, D.J., Kraft, N., Matsuzaki, I., Musso, D., Palinkas, L.A., Salnitskiy, V.P., Sipes, W., Stuster, J., \& Wang, J. (2009). Psychology and culture during long-duration space missions. Acta Astronautica, 64(7-8), 659-677. DOI

Kuznetsova, P., Gushin, V., Vinokhodova, A., \& Stepanova, A. (2018, October). Relationship between emotional stability, group status and cohesion in the international crew during simulated Mars exploration mission. Paper ID 42682 presented at the 69th International Astronautical Congress, Bremen, Germany.

Ma, Q. Sandal, G.M., Wu, R., Xiong, J, Xu, Z., He, L., Liu, Y., \& Li, Y. (2019). Personal value diversity in confinement and isolation: Pilot study results from the 180-day CELSS integration experiment. Acta Astronautica, 164, 84-91. DOI

Marboe, I. (2019). Living in the Moon village - Ethical and legal questions. Acta Astronautica, 154, 177-180. DOI

Marino, L. (2019). Humanity is not prepared to colonize Mars. Futures, 110, 15-18. DOI

Mittelstäedt, J.M., Pecena, Y., Outbaid, V., \& Maschke, P. (2016). Psychometric personality differences between candidates in astronaut selection. Aerospace Medicine and Human Performance, 87(11), 933-939. DOI

Palinkas, L.A. (2003). On the ICE: individual and group adaptation in Antarctica. Retrieved from http://www.bec.ucla.edu/papers/Palinkas_On_The_Ice.pdf

Polackova Solcovà, I., Lacev, A., \& Solcovà, I. (2014). Study of individual and group affective processes in the crew of a simulated mission to Mars: positive affectivity as a valuable indicator of changes in the crew affectivity. Acta Astronautica, 100, 57-67. DOI

Ritsher, J.B., Kanas, N.A., Ihle, E.C., \& Saylor, S.A. (2007). Psychological adaptation and salutogenesis in space: lessons from a series of studies. Acta Astronautica, 60(4-7), 336-340. $\underline{\text { DOI }}$

Sandal, G.M., Bye, H.H., \& Van de Vijver, F.J.R. (2011). Personal values and crew compatibility: results from a 105 days simulated space mission. Acta Astronautica, 69(3-4), 141-149. DOI

Sandal, G.M., Van DeVijver, F.J.R., \& Smith, N. (2018). Psychological hibernation in Antarctica. Frontiers in Psychology, 9, 2235. DOI

Schwartz, S.H., Cieciuch, J., Vecchione, M., Davidov, E., Fischer, R., Beierlein, C., Ramos, A., Verkasalo, M., Lönnqvist, J.E., Demirutku, K, Dirilen-Gumus, O., \& Konty, M., (2012). Refining the theory of basic individual values. Journal of Personality and Social Psychology, 103(4), 663-688. DOI 
Sharma, M. (2020). Covid-19 and its latest research trends: a brief analysis. International Journal of Recent Scientific Research, 11, 38676-38681.

Sherwood, B. (2017). Space architecture for MoonVillage. Acta Astonautica, 139, 396-406. DOI

Slobodian, R.E. (2015). Selling space colonization and immortality: a psychosocial, anthropological critique of the rush to colonize Mars. Acta Astronautica, 113, 89-104. DOI

Smith, C. (2016). An adaptive paradigm for human space settlement. Acta Astronautica, 119, 207-217. DOI

Smith, N., Sandal, G.M., Leon, G.R., \& Kjærgaard, K. (2017). Examining personal values in extreme environment contexts: revisiting the question of generalizability. Acta Astronautica, 137, 138-144. DOI

Solcovà, I., \& Tavel, P. (2017). Stress-related growth in two challenging conditions. Journal of Human Performance in Extreme Environments, 13(1), Article 4. DOI

Suedfeld, P., Brcic J., Johnson, P.J., \& Gushin, V. (2015). Coping strategies during and after spaceflight: data from retired cosmonauts. Acta Astronautica, 110, 43-49. DOI

Szocik, K. (2019). Should and could humans got to Mars? Yes, but not now and not in the near future. Futures, 105, 54-66. DOI

Szocik, K., Abood, S., Impey, C., Shelhamer M., Haqq-Mistra, J., Persson, E., Oviedo, L., Capova, K.A., Braddock, M., Rappaport, M.B., \& Corbally, C. (2020). Visions of a Martian future. Futures, 117, 102514. DOI

Tafforin, C. (2004). Ethological analysis of a polar team in the French Antarctic station Dumont d'Urville as simulation of space teams for future interplanetary missions. Acta Astronautica, 55(1), 51-60. DOI

Tafforin, C. (2005). Ethological indicators of isolated and confined teams in the perspective of Mars missions. Aviation Space and Environmental Medicine, 76, 1083-1087.

Tafforin, C. (2018). From the individual to the cultural space group. In T. Russomano \& L. Rehnberg (Eds.). IntoSpace: a journey of how humans adapt and live in microgravity (pp. 31-44). London: IntechOpen. DOI

Tafforin, C. (2020). Behaviors of a mixed gender and culture group during a 4-month confinement (SIRIUS-19). Antrocom Journal of Anthropology, 16, 5-19.

Van Wijk, C.H. (2018). Coping during conventional submarine missions: evidence of a third quarter phonomenon? Journal of Human Performance in Extreme Environments, 14(1), Article 12. DOI

Vermaa, S., Saharanb, A., Polcumpallyc, A.T., \& Biswasb, M. (2020). Tentacles of Covid-19 in India: impact on India Economy, Society, Polity and Geopolitics. Journal of Humanities and Social Science Studies, 2, 54-61.

Vinokhodova, A.G., \& Gushin, V.I. (2014). Study of values and interpersonal perception in cosmonauts on board of International Space Station. Acta Astronautica, 93, 359-365. DOI

Wang, Y., \& Wu, R. (2015). Time effects, displacement and leadership roles on a Lunar space station analogue. Aerospace Medicine and Human Performance, 86(9), 819-823. DOI

Wooster, P.D., Braun, R.D., Ahn, J., Putnam, Z.R. (2007). Mission design options for human Mars missions. Mars - The International Journal of Mars Science and Exploration, 3, 12-28. DOI

Wu, R., \& Wang, Y. (2015). Psychosocial interaction during a 105-day isolated mission in Lunar Palace 1. Acta Astronautica, 113, 1-7. DOI 